\title{
A new generalization of metric spaces: rectangular $M$-metric spaces
}

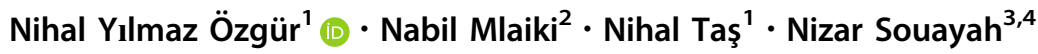

Received: 28 February 2018 / Accepted: 3 September 2018/Published online: 17 September 2018

(C) The Author(s) 2018

\begin{abstract}
In this paper, we introduce the concept of the rectangular $M$-metric spaces, along with its topology and we prove some fixed-point theorems under different contraction principles with various techniques. The obtained results generalize some classical fixed-point results such as the Banach's contraction principle, the Kannan's fixed-point theorem and the Chatterjea's fixed-point theorem. Also we give an application to the fixed-circle problem.
\end{abstract}

Keywords Rectangular $M$-metric space $\cdot$ Fixed point

Mathematics Subject Classification Primary 54E35 - Secondary 54E40 $\cdot 54 \mathrm{H} 25 \cdot 47 \mathrm{H} 10$

\section{Introduction}

The well-known Banach contraction principle has been studied and generalized in many different directions such as generalizing the used metric spaces. Recently, new generalized metric spaces have been presented for this purpose. For example, $M$-metric spaces, rectangular metric spaces, partial rectangular metric spaces have been introduced and studied (see [2, 3, 7]). Branciari in [3] defined rectangular metric spaces as follows:

Definition 1.1 [3] (Rectangular metric space (Branciari metric space)) Let $X$ be a nonempty set. A mapping $d$ : $X \times X \rightarrow \mathbb{R}^{+}$is said to be a rectangular metric on $X$ if for

Nihal Yllmaz Özgür

nihal@balikesir.edu.tr

Nabil Mlaiki

nmlaiki@psu.edu.sa

Nihal Taş

nihaltas@balikesir.edu.tr

Nizar Souayah

nizar.souayah@yahoo.fr

1 Department of Mathematics, Balikesir University, 10145 Balikesir, Turkey

2 Department of Mathematical Sciences, Prince Sultan University, Riyadh, Saudi Arabia

3 Department of Natural Sciences, Community College ALRiyadh, King Saud University, Riyadh, Saudi Arabia

4 ESSECT, University of Tunis, Tunis, Tunisia any $x, y \in X$ and all distinct points $u, v \in X \backslash\{x, y\}$, it satisfies the following conditions:

$\left(R_{1}\right) \quad x=y$ if and only if $d(x, y)=0$;

$\left(R_{2}\right) \quad d(x, y)=d(y, x)$

$\left(R_{3}\right) \quad d(x, y) \leq d(x, u)+d(u, v)+d(v, y)$ (rectangular inequality).

In this case the pair $(X, d)$ is called a rectangular metric space.

Inspired by the work of Branciari, Shukla in [7] defined rectangular partial metric spaces which are generalizations of rectangular metric spaces.

Definition 1.2 [7] (Partial rectangular metric space) Let $X$ be a nonempty set. A mapping $\rho: X \times X \rightarrow \mathbb{R}^{+}$is said to be a partial rectangular metric on $X$ if for any $x, y \in X$ and all distinct points $u, v \in X \backslash\{x, y\}$, it satisfies the following conditions :

$$
\begin{array}{ll}
\left(R P_{1}\right) & x=y \text { if and only if } \rho(x, y)=\rho(x, x)=\rho(y, y) \\
\left(R P_{2}\right) & \rho(x, x) \leq \rho(x, y) \\
\left(R P_{3}\right) & \rho(x, y)=\rho(y, x) \\
\left(R P_{4}\right) & \rho(x, y) \leq \rho(x, u)+\rho(u, v)+\rho(v, y) \\
& -\rho(u, u)-\rho(v, v)
\end{array}
$$

In this case, the pair $(X, \rho)$ is called a partial rectangular metric space.

Asadi et al. in [2] gave an extension to the partial metric spaces, called $M$-metric spaces, defined as follows. 


\section{Notation 1.3 [2]}

1. $m_{x, y}:=\min \{m(x, x), m(y, y)\}$.

2. $M_{x, y}:=\max \{m(x, x), m(y, y)\}$.

Definition 1.4 [2] Let $X$ be a nonempty set. If the function $m: X \times X \rightarrow \mathbb{R}^{+}$satisfies the following conditions for all $x, y, z \in X$

$\left(M_{1}\right) \quad m(x, x)=m(y, y)=m(x, y)$ if and only if $x=y$,

$\left(M_{2}\right) \quad m_{x, y} \leq m(x, y)$,

$\left(M_{3}\right) \quad m(x, y)=m(y, x)$,

$\left(M_{4}\right) \quad\left(m(x, y)-m_{x, y}\right) \leq\left(m(x, z)-m_{x, z}\right)$

$+\left(m(z, y)-m_{z, y}\right)$,

then the pair $(X, m)$ is called an $M$-metric space.

On these new spaces, some generalized fixed-point results have been obtained (see $[1-3,6,7]$ ). In this paper, we introduce the concept of a rectangular $M$-metric space, along with proving some fixed-point theorems for selfmappings in rectangular $M$-metric spaces. In Sect. 2, we define the notion of a rectangular $M$-metric space and investigate some basic properties of this new space. In Sect. 3, we present some topological concepts about open balls and convergence in rectangular $M$-metric spaces. In Sect. 4, we prove new generalizations of classical fixedpoint results such as the Banach's contraction principle, the Kannan's fixed-point theorem and the Chatterjea's fixedpoint theorem. In Sect. 5, we define the notions of a circle and a fixed circle. Using these concepts, we present an application to fixed-circle problem.

\section{Rectangular $\boldsymbol{M}$-metric spaces}

At first, we need to present the following notation.

\section{Notation 2.1}

1. $m_{r_{x, y}}:=\min \left\{m_{r}(x, x), m_{r}(y, y)\right\}$.

2. $M_{r_{x, y}}:=\max \left\{m_{r}(x, x), m_{r}(y, y)\right\}$.

Definition 2.2 Let $X$ be a nonempty set and $m_{r}: X \times X \rightarrow$ $[0, \infty)$ be a function. If the following conditions are satisfied for all $x, y$ in $X$

$$
\begin{array}{ll}
\left(R M_{1}\right) & m_{r}(x, y)=m_{r_{x, y}}=M_{r_{x, y}} \Longleftrightarrow x=y, \\
\left(R M_{2}\right) & m_{r_{x, y}} \leq m_{r}(x, y), \\
\left(R M_{3}\right) & m_{r}(x, y)=m_{r}(y, x), \\
\left(R M_{4}\right) & m_{r}(x, y)-m_{r_{x, y}} \leq m_{r}(x, u)-m_{r_{x, u}}+m_{r}(u, v) \\
& -m_{r_{u, v}}+m_{r}(v, y)-m_{r_{v, y}} \text { for all } u, v \in X \backslash\{x, y\},
\end{array}
$$

then the pair $\left(X, m_{r}\right)$ is called a rectangular $M$-metric space.

Notice that every $M$-metric is also a rectangular $M$ metric.

Remark 2.3 Let $\left(X, m_{r}\right)$ be a rectangular $M$-metric space. Clearly, we have

(1) $0 \leq M_{r_{x, y}}+m_{r_{x, y}}=m_{r}(x, x)+m_{r}(y, y)$,

(2) $0 \leq M_{r_{x, y}}-m_{r_{x, y}}=\left|m_{r}(x, x)-m_{r}(y, y)\right|$

for every $x, y \in X$.

Also it can be easily verified the following inequality under some cases:

$$
\begin{aligned}
& M_{r_{x, y}}-m_{r_{x, y}} \leq\left(M_{r_{x, u}}-m_{r_{x, u}}\right)+\left(M_{r_{u, v}}-m_{r_{u, v}}\right) \\
& +\left(M_{r_{v, y}}-m_{r_{v, y}}\right) .
\end{aligned}
$$

For example, if we consider the case

$m_{r}(x, x) \geq m_{r}(u, u) \geq m_{r}(v, v) \geq m_{r}(y, y)$,

then we get

$$
\begin{aligned}
M_{r_{x, y}}-m_{r_{x, y}=}= & m_{r}(x, x)-m_{r}(y, y) \\
= & m_{r}(x, x)-m_{r}(y, y)+m_{r}(u, u)-m_{r}(u, u) \\
& +m_{r}(v, v)-m_{r}(v, v) \\
= & \left(m_{r}(x, x)-m_{r}(u, u)\right)+\left(m_{r}(u, u)-m_{r}(v, v)\right) \\
& +\left(m_{r}(v, v)-m_{r}(y, y)\right) \\
= & \left(M_{r_{x, u}}-m_{r_{x, u}}\right)+\left(M_{r_{u, v}}-m_{r_{u, v}}\right)+\left(M_{r_{v, y}}-m_{r_{v, y}}\right) .
\end{aligned}
$$

Now we give some examples.

Example 2.4 Let $\mathbb{C}$ be the set of all complex numbers, and consider the set $X_{\theta}=\{z \in \mathbb{C}: \arg (z)=\theta\} \cup\{0\}$ for a fixed $\theta, 0 \leq \theta<2 \pi$. If we define the self-mapping $m_{r}$ on $X_{\theta}$ given by $m_{r}(x, y)=\frac{|x|+|y|}{2}$ for all $x, y \in X_{\theta}$, then $\left(X_{\theta}, m_{r}\right)$ is a rectangular $M$-metric space.

We will only show that the following triangular inequality holds since the other conditions of the metric are satisfied (easy to check).

$$
\begin{aligned}
& m_{r}(x, y)-m_{r_{x, y}} \leq m_{r}(x, u)-m_{r_{x, u}}+m_{r}(u, v)-m_{r_{u, v}} \\
& \quad+m_{r}(v, y)-m_{r_{v, y} .}
\end{aligned}
$$

Let $x, y, u, v \in X_{\theta}$. We suppose without loss of generality that $|x| \leq|y|$. Then, $m_{r}(x, y)=\frac{|x|+|y|}{2}, m_{r}(x, u)=\frac{|x|+|u|}{2}$, $m_{r}(u, v)=\frac{|u|+|v|}{2}$. We need to consider the following cases: 
Case 1: $\quad|u| \leq|v| \leq|x| \leq|y|$ :

We have $m_{r_{x, y}}=|x|, m_{r_{x, u}}=|u|, m_{r_{u, v}}=|u|$ and $m_{r_{v, y}}=|v|$. Therefore, (2.1) holds. Indeed, (2.1) can be written as follows:

$$
\begin{aligned}
& \frac{|x|+|y|}{2}-|x| \leq \frac{|x|+|u|}{2}-|u|+\frac{|u|+|v|}{2}-|u| \\
& +\frac{|v|+|y|}{2}-|v|=\frac{|x|+|y|}{2}+|u|+|v| \\
& -|u|-|u|-|v|=\frac{|x|+|y|}{2}-|u|,
\end{aligned}
$$

which is correct since $|u| \leq|x|$.

Case 2: $\quad|u| \leq|x| \leq|v| \leq|y|$ : It follows as in the previous case.

Case 3: $\quad|u| \leq|x| \leq|y| \leq|v|$ :

We obtain

$$
\begin{aligned}
\frac{|x|+|y|}{2}-|x| & \leq \frac{|x|+|u|}{2}-|u|+\frac{|u|+|v|}{2}-|u| \\
& +\frac{|v|+|y|}{2}-|y| \\
& =\frac{|x|+|y|}{2}-(|u|+|y|-|v|),
\end{aligned}
$$

which is correct given that $|u|+|y|-|v|<|x|$.

Case 4: $\quad|x| \leq|u| \leq|v| \leq|y|$ :

$$
\begin{aligned}
\frac{|x|+|y|}{2}-|x| & \leq \frac{|x|+|u|}{2}-|u|+\frac{|u|+|v|}{2} \\
& -|u|+\frac{|v|+|y|}{2}-|v|=\frac{|x|+|y|}{2} \\
& +|u|+|v|-|u|-|u|-|v| \\
& \leq \frac{|x|+|y|}{2}-|x| .
\end{aligned}
$$

Case 5: $\quad|x| \leq|u| \leq|y| \leq|v|$ :

$$
\begin{aligned}
\frac{|x|+|y|}{2}-|x| & \leq \frac{|x|+|u|}{2}-|x|+\frac{|u|+|v|}{2}-|u| \\
& +\frac{|v|+|y|}{2}-|y|=\frac{|x|+|y|}{2}+|u| \\
& +|v|-|x|-|u|-|y| \\
& =\frac{|x|+|y|}{2}-(|x|+|y|-|v|) .
\end{aligned}
$$

Since $|x|+|y|-|v| \leq|x|$, therefore the inequality holds.

Case 6: $\quad|x| \leq|y| \leq|u| \leq|v|$ : It follows as in the previous case.
We note that if we permute $u$ and $v$ in all the precedent cases, (2.1) is still valid. Hence, $\left(X_{\theta}, m_{r}\right)$ is a rectangular $M$-metric space.

Proposition 2.5 Let $(X, d)$ be a rectangular metric space and a function $\xi:[0, \infty) \rightarrow[\alpha, \infty)$ be a one-to-one and nondecreasing function with $\xi(0)=\alpha$ such that

$\xi(x+y+z) \leq \xi(x)+\xi(y)+\xi(z)-2 \alpha$,

for all $x, y, z \in[0, \infty)$. Then, the function $m_{r}: X \times X \rightarrow$ $[0, \infty)$ is defined as

$m_{r}(x, y)=\xi(d(x, y))$,

for all $x, y \in X$ is a rectangular $M$-metric.

Proof From the hypothesis, it can be easily checked that the conditions $\left(R M_{1}\right),\left(R M_{2}\right)$ and $\left(R M_{3}\right)$ are satisfied. Now we show that the condition $\left(R M_{4}\right)$ is satisfied. Using the condition $\left(R_{3}\right)$, we obtain

$$
\begin{aligned}
\xi(d(x, y)) & \leq \xi(d(x, u)+d(u, v)+d(v, y)) \\
& \leq \xi(d(x, u))+\xi(d(u, v))+\xi(d(v, y))-2 \alpha
\end{aligned}
$$

and

$$
\begin{aligned}
\xi(d(x, y))-\alpha & \leq(\xi(d(x, u))-\alpha)+(\xi(d(u, v))-\alpha) \\
& +(\xi(d(v, y))-\alpha) .
\end{aligned}
$$

Therefore, we get

$$
\begin{aligned}
m_{r}(x, y)-m_{r_{x, y}} & \leq m_{r}(x, u)-m_{r_{x, u}}+m_{r}(u, v)-m_{r_{u, v}} \\
& +m_{r}(v, y)-m_{r_{v, y} .}
\end{aligned}
$$

Consequently, $m_{r}$ is a rectangular $M$-metric.

Example 2.6 Let $(X, d)$ be a rectangular metric space and a function $\xi:[0, \infty) \rightarrow[\alpha, \infty)$ be defined as

$\xi(t)=m t+n$,

with $\xi(0)=\alpha$ for all $t \in[0, \infty)$. From Proposition 2.5, the function $m_{r}(x, y)=m d(x, y)+n$ is a rectangular $M$-metric.

Note that we can obtain a rectangular metric space from a rectangular $M$-metric space as seen in the following examples.

Example 2.7 Let $\left(X, m_{r}\right)$ be a rectangular $M$-metric space and $m_{r}^{w}: X \times X \rightarrow[0, \infty)$ be a function defined as

$m_{r}^{w}(x, y)=m_{r}(x, y)-2 m_{r_{x, y}}+M_{r_{x, y}}$,

for all $x, y \in X$. Then, $m_{r}^{w}$ is a rectangular metric and the pair $\left(X, m_{r}^{w}\right)$ is a rectangular metric space.

Now we show that the conditions $\left(R_{1}\right),\left(R_{2}\right)$ and $\left(R_{3}\right)$ are satisfied as follows:

$\left(R_{1}\right)$ Using the conditions $\left(R M_{1}\right)$ and $\left(R M_{2}\right)$, we get 


$$
\begin{aligned}
m_{r}^{w}(x, y) & =0 \Leftrightarrow m_{r}(x, y)-2 m_{r_{x, y}}+M_{r_{x, y}}=0 \\
& \Leftrightarrow m_{r}(x, y)=2 m_{r_{x, y}}-M_{r_{x, y}}
\end{aligned}
$$

and

$$
\begin{aligned}
m_{r_{x, y}} \leq m_{r}(x, y) & =2 m_{r_{x, y}}-M_{r_{x, y}} \Leftrightarrow M_{r_{x, y}} \leq m_{r_{x, y}} \Leftrightarrow M_{r_{x, y}}=m_{r_{x, y}} \\
\Leftrightarrow & \Leftrightarrow m_{r}(x, y)=m_{r}(x, x)=m_{r}(y, y) \Leftrightarrow x=y,
\end{aligned}
$$

for all $x, y \in X$.

$\left(R_{2}\right)$ Using the conditions $\left(R M_{3}\right)$, we have

$$
\begin{aligned}
m_{r}^{w}(x, y) & =m_{r}(x, y)-2 m_{r_{x, y}}+M_{r_{x, y}} \\
& =m_{r}(y, x)-2 m_{r_{y, x}}+M_{r_{y, x}}=m_{r}^{w}(y, x),
\end{aligned}
$$

for all $x, y \in X$.

$\left(R_{3}\right)$ Using the conditions $\left(R M_{4}\right)$ and the inequality (3) given in Remark 2.3, we get

$$
\begin{aligned}
m_{r}^{w}(x, y)= & m_{r}(x, y)-2 m_{r_{x, y}}+M_{r_{x, y}} \\
= & \left(m_{r}(x, y)-m_{r_{x, y}}\right)+\left(M_{r_{x, y}}-m_{r_{x, y}}\right) \\
\leq & {\left[m_{r}(x, u)-m_{r_{x, u}}+m_{r}(u, v)-m_{r_{u, v}}+m_{r}(v, y)-m_{r_{v, y}}\right] } \\
& +\left[\left(M_{r_{x, u}}-m_{r_{x, u}}\right)+\left(M_{r_{u, v}}-m_{r_{u, v}}\right)+\left(M_{r_{v, y}}-m_{r_{v, y}}\right)\right] \\
= & m_{r}^{w}(x, u)+m_{r}^{w}(u, v)+m_{r}^{w}(v, y),
\end{aligned}
$$

for all $u, v \in X \backslash\{x, y\}$. Consequently, $\left(X, m_{r}^{w}\right)$ is a rectangular metric space.

Example 2.8 Let $\left(X, m_{r}\right)$ be a rectangular $M$-metric space and $m_{r}^{s}: X \times X \rightarrow[0, \infty)$ be a function defined as

$m_{r}^{s}(x, y)=m_{r}(x, y)-m_{r_{x, y}}$,

for all $x, y \in X$ such that if $m_{r}^{s}(x, y)=0$ then $x=y$. Then, $m_{r}^{s}$ is a rectangular metric and the pair $\left(X, m_{r}^{s}\right)$ is a rectangular metric space.

Now we show that the conditions $\left(R_{1}\right),\left(R_{2}\right)$ and $\left(R_{3}\right)$ are satisfied as follows :

$\left(R_{1}\right)$ Using the hypothesis and the definition of $m_{r}^{s}$, we get

$$
\begin{aligned}
x & =y \Rightarrow m_{r}^{s}(x, x)=m_{r}(x, x)-m_{r_{x, x}} \\
& =m_{r}(x, x)-\min \left\{m_{r}(x, x), m_{r}(x, x)\right\}=0
\end{aligned}
$$

and

$m_{r}^{s}(x, y)=0 \Rightarrow x=y$,

for all $x, y \in X$.

$\left(R_{2}\right)$ Using the condition $\left(R M_{3}\right)$, we have

$m_{r}^{s}(x, y)=m_{r}(x, y)-m_{r_{x, y}}=m_{r}(y, x)-m_{r_{y, x}}=m_{r}^{s}(y, x)$,

for all $x, y \in X$.

$\left(R_{3}\right)$ Using the condition $\left(R M_{4}\right)$, we obtain

$$
\begin{aligned}
m_{r}^{s}(x, y) & =m_{r}(x, y)-m_{r_{x, y}} \\
& \leq m_{r}(x, u)-m_{r_{x, u}}+m_{r}(u, v)-m_{r_{u, v}}+m_{r}(v, y)-m_{r_{v, y}} \\
& =m_{r}^{s}(x, u)+m_{r}^{s}(u, v)+m_{r}^{s}(v, y),
\end{aligned}
$$

for all $u, v \in X \backslash\{x, y\}$. Consequently, $\left(X, m_{r}^{s}\right)$ is a rectangular metric space.

In the following proposition, we see the relationship between a rectangular partial metric and a rectangular $M$ metric.

Proposition 2.9 Every partial rectangular metric is a rectangular M-metric.

Proof Let $m_{r}$ be a partial rectangular metric. Let us consider the following cases:

$$
\begin{aligned}
& m_{r}(x, x)=m_{r}(y, y)=m_{r}(u, u)=m_{r}(v, v), \\
& m_{r}(x, x)<m_{r}(y, y)<m_{r}(u, u)<m_{r}(v, v), \\
& m_{r}(x, x)=m_{r}(y, y)=m_{r}(u, u)<m_{r}(v, v), \\
& m_{r}(x, x)=m_{r}(y, y)<m_{r}(u, u)<m_{r}(v, v), \\
& m_{r}(x, x)=m_{r}(y, y)<m_{r}(u, u)<m_{r}(v, v), \\
& m_{r}(x, x)<m_{r}(y, y)<m_{r}(u, u)=m_{r}(v, v), \\
& m_{r}(x, x)<m_{r}(y, y)=m_{r}(u, u)<m_{r}(v, v), \\
& m_{r}(x, x)<m_{r}(y, y)=m_{r}(u, u)=m_{r}(v, v), \\
& m_{r}(x, x)>m_{r}(y, y)>m_{r}(u, u)>m_{r}(v, v), \\
& m_{r}(x, x)=m_{r}(y, y)=m_{r}(u, u)>m_{r}(v, v), \\
& m_{r}(x, x)=m_{r}(y, y)>m_{r}(u, u)=m_{r}(v, v), \\
& m_{r}(x, x)=m_{r}(y, y)>m_{r}(u, u)>m_{r}(v, v), \\
& m_{r}(x, x)>m_{r}(y, y)>m_{r}(u, u)=m_{r}(v, v), \\
& m_{r}(x, x)>m_{r}(y, y)=m_{r}(u, u)>m_{r}(v, v), \\
& m_{r}(x, x)>m_{r}(y, y)=m_{r}(u, u)=m_{r}(v, v) .
\end{aligned}
$$

Under the above cases, the condition $\left(R M_{4}\right)$ is satisfied. For example, if we consider case (2), then we get

$$
\begin{aligned}
& m_{r}(x, y) \leq m_{r}(x, u)+m_{r}(u, v)+m_{r}(v, y)-m_{r}(u, u) \\
& \quad-m_{r}(u, v)
\end{aligned}
$$

and so

$$
\begin{aligned}
m_{r}(x, y)-m_{r x, y}= & m_{r}(x, y)-m_{r}(x, x) \\
\leq & m_{r}(x, u)+m_{r}(u, v)+m_{r}(v, y) \\
& -m_{r}(u, u)-m_{r}(v, v)-m_{r}(x, x) \\
\leq & {\left[m_{r}(x, u)-m_{r}(x, x)\right]+\left[m_{r}(u, v)\right.} \\
& \left.-m_{r}(u, u)\right]+\left[m_{r}(v, y)-m_{r}(y, y)\right] \\
& =m_{r}(x, y)-m_{r_{x, y}}+m_{r}(u, v)-m_{r_{u, v}} \\
& +m_{r}(v, y)-m_{r_{v, y}},
\end{aligned}
$$

for all $u, v \in X \backslash\{x, y\}$. Using the similar arguments, it can be easily seen that the condition $\left(R M_{4}\right)$ is satisfied under the other cases. Therefore, the partial rectangular metric $m_{r}$ is a rectangular $M$-metric.

The converse statement of Proposition 2.9 is not always true as seen in the following example.

Example 2.10 Let $X=\{1,2,3,4\}$ and the function $m_{r}$ : $X \times X \rightarrow[0, \infty)$ be defined by 


$$
\begin{gathered}
m_{r}(1,1)=m_{r}(2,2)=m_{r}(3,3)=1 \operatorname{andm}_{\mathrm{r}}(4,4)=8, \\
m_{r}(1,2)=m_{r}(2,1)=4, \\
m_{r}(1,3)=m_{r}(3,1)=4 \\
m_{r}(1,4)=m_{r}(4,1)=4 \\
m_{r}(2,3)=m_{r}(3,2)=5 \\
m_{r}(2,4)=m_{r}(4,2)=6 \\
m_{r}(3,4)=m_{r}(4,3)=7
\end{gathered}
$$

for all $x, y \in X$. Then, $m_{r}$ is a rectangular $M$-metric, but it is not a rectangular partial metric on $X$. Indeed, for $x=4$, $y=3$, we have

$m_{r}(4,4)=8 \leq m_{r}(4,3)=7$,

which is a contradiction. Therefore, the condition $\left(R P_{2}\right)$ is not satisfied.

It is known that every metric space is a rectangular metric space (see [4]) and that every rectangular metric space is a partial rectangular metric space with zero selfdistance (see [7]). Also every metric space is a partial metric space and every partial metric space is an $M$-metric space (see $[2,5])$. Consequently, we can give the following diagram. Here, arrows stand for inclusions.
(3) A rectangular $M$-metric space is said to be $m_{r}$ complete if every $m_{r}$-Cauchy sequence $\left\{x_{n}\right\}$ converges to a point $x$ such that

$$
\begin{aligned}
\lim _{n \rightarrow \infty}\left(m_{r}\left(x_{n}, x\right)-m_{r_{x_{n}, x}}\right) & =0 \text { and } \lim _{\mathrm{n} \rightarrow \infty}\left(\mathrm{M}_{\mathrm{r}_{\mathrm{x}, \mathrm{x}}}-\mathrm{m}_{\mathrm{r}_{\mathrm{x}, \mathrm{x}}}\right) \\
& =0 .
\end{aligned}
$$

Lemma 3.2 Assume that $x_{n} \longrightarrow x$ and $y_{n} \longrightarrow y$ as $n \longrightarrow$ $\infty$ in a rectangular $M$-metric space $\left(X, m_{r}\right)$. Then,

$\lim _{n \rightarrow \infty}\left(m_{r}\left(x_{n}, y_{n}\right)-m_{r_{x_{n}, y_{n}}}\right)=m_{r}(x, y)-m_{r_{x, y}}$.

Proof Using the triangular inequality of the rectangular $M$-metric, we obtain

$$
\begin{aligned}
& m_{r}\left(x_{n}, y_{n}\right)-m_{x_{x_{n}, y_{n}}} \leq m_{r}\left(x_{n}, x\right)-m_{r_{x_{n}, x}}+m_{r}(x, y)-m_{r_{x, y}} \\
& \quad+m_{r}\left(y, y_{n}\right)-m_{r_{y, y_{n}} .}
\end{aligned}
$$

Then,

$$
\begin{aligned}
& m_{r}\left(x_{n}, y_{n}\right)-m_{x_{x_{n}, y_{n}}}-m_{r}(x, y)+m_{r_{x, y}} \leq m_{r}\left(x_{n}, x\right)-m_{r_{x_{n}, x}} \\
& \quad+m_{r}\left(y, y_{n}\right)-m_{r_{y, y_{n}}} .
\end{aligned}
$$

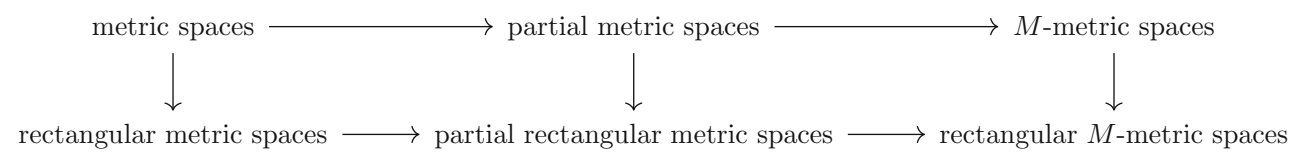

\section{Some topological notions of rectangular $M$ - metric spaces}

In this section, we investigate some topological properties of rectangular $M$-metric spaces.

\section{Convergence in rectangular $M$-metric spaces}

Definition 3.1 Let $\left(X, m_{r}\right)$ be a rectangular $M$-metric space. Then, we have

(1) A sequence $\left\{x_{n}\right\}$ in $X$ converges to a point $x$ if and only if

$\lim _{n \rightarrow \infty}\left(m_{r}\left(x_{n}, x\right)-m_{r_{x_{n}, x}}\right)=0$.

(2) A sequence $\left\{x_{n}\right\}$ in $X$ is said to be $m_{r}$-Cauchy sequence if and only if

$\lim _{n, m \rightarrow \infty}\left(m_{r}\left(x_{n}, x_{m}\right)-m_{r_{x_{n}, x_{m}}}\right)$ and $\lim _{\mathrm{n}, \mathrm{m} \rightarrow \infty}\left(\mathrm{M}_{\mathrm{r}_{\mathrm{x}}, x_{\mathrm{m}}}-\mathrm{m}_{\mathrm{r}_{\mathrm{x}_{\mathrm{n}}, x_{\mathrm{m}}}}\right)$

exist and finite.
Knowing that $\left(x_{n}\right)$ converges to $x$ and $\left(y_{n}\right)$ converges to $y$, we obtain the result from (3.2), that is,

$$
\begin{aligned}
& m_{r}(x, y)-m_{r_{x, y}} \leq m_{r}\left(x, x_{n}\right)-m_{r_{x, x_{n}}}+m_{r}\left(x_{n}, y_{n}\right)-m_{r_{x_{n}, y_{n}}} \\
& \quad+m_{r}\left(y_{n}, y\right)-m_{r_{y_{n}, y}}
\end{aligned}
$$

and then

$m_{r}(x, y)-m_{r_{x, y}} \leq \lim _{n \rightarrow \infty}\left(m_{r}\left(x_{n}, y_{n}\right)-m_{r_{x_{n}, y_{n}}}\right)$.

From Lemma 3.2, we can deduce the following lemma.

Lemma 3.3 Assume that $x_{n} \longrightarrow x$ as $n \longrightarrow \infty$ in a rectangular M-metric space $\left(X, m_{r}\right)$. Then

$\lim _{n \rightarrow \infty}\left(m_{r}\left(x_{n}, y\right)-m_{x_{x_{n}, y}}\right)=m_{r}(x, y)-m_{r_{x, y}}$ for all $y \in X$.

Lemma 3.4 Assume that $x_{n} \longrightarrow x$ and $y_{n} \longrightarrow y$ as $n \longrightarrow$ $\infty$ in a rectangular $M$-metric space $\left(X, m_{r}\right)$. Then, $m_{r}(x, y)=m_{r_{x, y}}$. Further if $m_{r}(x, x)=m_{r}(y, y)$, then $x=y$. 
Proof From Lemma 3.2, we have

$0=\lim _{n \rightarrow \infty}\left(m_{r}\left(x_{n}, y_{n}\right)-m_{r_{x_{n}, y_{n}}}\right)=m_{r}(x, y)-m_{r_{x, y}}$

and then

$m_{r}(x, y)=m_{r_{x, y}}$.

From the condition $\left(R M_{1}\right)$ and the hypothesis $m_{r}(x, x)=m_{r}(y, y)$, we get $x=y$.

Lemma 3.5 Let $\left\{x_{n}\right\}$ be a sequence in a rectangular $M$ metric space $\left(X, m_{r}\right)$, such that

there exists $r \in[0,1)$ such that $m_{r}\left(x_{n+1}, x_{n}\right) \leq r m_{r}\left(x_{n}, x_{n-1}\right)$ for all $n \in \mathbb{N}$.

Then,
(A) $\lim _{n \rightarrow \infty} m_{r}\left(x_{n}, x_{n-1}\right)=0$,
(B) $\lim _{n \rightarrow \infty} m_{r}\left(x_{n}, x_{n}\right)=0$,
(C) $\lim _{n, m \rightarrow \infty} m_{r_{x_{n}, x_{m}}}=0$,
(D) $\left\{x_{n}\right\}$ is an $m_{r}$-Cauchy sequence.

Proof Using the definition of convergence and inequality (3.4), the proof of the condition (A) follows easily. From the condition $\left(R M_{2}\right)$ and the condition $(\mathrm{A})$, we get

$$
\begin{aligned}
& \lim _{n \rightarrow \infty} \min \left\{m_{r}\left(x_{n}, x_{n}\right), m_{r}\left(x_{n-1}, x_{n-1}\right)\right\}=\lim _{n \rightarrow \infty} m_{r_{x_{n}, x_{n-1}}} \\
& \quad \leq \lim _{n \rightarrow \infty} m_{r}\left(x_{n}, x_{n-1}\right)=0 .
\end{aligned}
$$

Therefore, the condition (B) holds. Since $\lim _{n \rightarrow \infty} m_{r}\left(x_{n}, x_{n}\right)=0$, the condition (C) holds. Using the previous conditions and Definition 3.1, we see that the condition (D) holds.

Lemma 3.6 Let $\left(X, m_{r}\right)$ be a rectangular $M$-metric space. Then, we get

(1) $\left\{x_{n}\right\}$ is an $m_{r}$-Cauchy sequence in $\left(X, m_{r}\right)$ if and only if $\left\{x_{n}\right\}$ is a Cauchy sequence in $\left(X, m_{r}^{w}\right)$ (resp. $\left.\left(X, m_{r}^{s}\right)\right)$.

(2) $\left(X, m_{r}\right)$ is $m_{r}$-complete if and only if $\left(X, m_{r}^{w}\right)$ ( resp. $\left.\left(X, m_{r}^{s}\right)\right)$ is complete.

Proof Using Examples 2.7 and 2.8, the proof follows easily.

\section{Topology of rectangular $M$-metric spaces}

Let $m_{r}$ be a rectangular $M$-metric on $X$. For all $x \in X$ and $\varepsilon>0$, the open ball with the center $x$ and the radius $\varepsilon$ is $B(x, \varepsilon)=\left\{y \in X: m_{r}(x, y)-m_{r_{x, y}}<\varepsilon\right\}$.

Notice that we have $x \in B(x, \varepsilon)$ for all $\varepsilon>0$. Indeed, we get $m_{r}(x, x)-m_{r_{x, x}}=m_{r}(x, x)-m_{r}(x, x)=0<\varepsilon$.

Similarly, the closed ball with the center $x$ and the radius $\varepsilon$ is

$B[x, \varepsilon]=\left\{y \in X: m_{r}(x, y)-m_{r_{x, y}} \leq \varepsilon\right\}$.

Lemma 3.7 Let $m_{r}$ be a rectangular M-metric on $X$. The collection of all open balls on $X$

$\mathcal{B}_{m_{r}}=\{B(x, \varepsilon)\}_{x \in X}^{\varepsilon>0}$,

forms a basis on $X$.

Proof Let $y \in B(x, \varepsilon)$. Then, we have

$m_{r}(x, y)-m_{r_{x, y}}<\varepsilon$,

for all $x \in X$ and $\varepsilon>0$. If we take

$\delta=\varepsilon-m_{r}(x, y)+m_{r_{x, y}}$,

then we get $\delta>0$. Now we show that

$B(y, \delta) \subseteq B(x, \varepsilon)$.

Let $z \in B(y, \delta)$. Then, we obtain

$m_{r}(y, z)-m_{r_{y, z}}<\delta$.

From the conditions $\left(R M_{4}\right),(3.5)$ and (3.6), we get

$$
\begin{aligned}
& m_{r}(x, z)-m_{r_{x, z}} \leq m_{r}(x, y)-m_{r_{x, y}}+m_{r}(y, y)-m_{r_{y, y}} \\
& +m_{r}(y, z)-m_{r_{y, z}}<\varepsilon-\delta+\delta=\varepsilon .
\end{aligned}
$$

Consequently, we find $B(y, \delta) \subseteq B(x, \varepsilon)$ and $\mathcal{B}_{m_{r}}$ is a basis on $X$.

\section{Definition 3.8}

a) Let $m_{r}$ be a rectangular $M$-metric on $X$ and $\tau_{m_{r}}$ be the topology generated by the open balls $B(x, \varepsilon)$. Then, the pair $\left(X, \tau_{m_{r}}\right)$ is called a rectangular $M$-space.

b) Let $\left(X, \tau_{m_{r}}\right)$ be a rectangular $M$ - space. $\left(X, \tau_{m_{r}}\right)$ is called a $T_{0}$-space if for any distinct pair of points $x, y \in X$, there exists an open ball containing $x$ but not $y$ or an open ball containing $y$ but not $x$.

Theorem 3.9 A rectangular $M$-space is a $T_{0}$-space.

Proof Let $\left(X, \tau_{m_{r}}\right)$ be a rectangular $M$-space and $x, y \in X$ with $x \neq y$. Without loss of generality, let us consider the following cases:

Case 1 If $m_{r}(x, x)=m_{r}(y, y)$, then using the hypothesis, the conditions $\left(R M_{1}\right)$ and $\left(R M_{2}\right)$, we get

$m_{r_{x, y}}=m_{r}(x, x)=m_{r}(y, y)<m_{r}(x, y)$

and

$m_{r}(x, y)-m_{r_{x, y}}=m_{r}(x, y)-m_{r}(x, x)>0$. 
Hence, if we take $\varepsilon=m_{r}(x, y)-m_{r}(x, x)$, then we obtain $y \notin B(x, \varepsilon)$.

Case 2 If $m_{r}(x, x)<m_{r}(y, y)$, then using the conditions $\left(R M_{1}\right)$ and $\left(R M_{2}\right)$, we get

$m_{r}(x, y)-m_{r_{x, y}}>0$

and

$m_{r}(x, y)-m_{r_{x, y}}=m_{r}(x, y)-m_{r}(x, x)>0$.

Hence, if we take $\varepsilon=m_{r}(x, y)-m_{r}(x, x)$, then we obtain $y \notin B(x, \varepsilon)$.

Consequently, $\left(X, \tau_{m_{r}}\right)$ is a $T_{0}$-space.

\section{Some fixed-point results}

At first, we prove the following useful lemma.

Lemma 4.1 Let $\left(X, m_{r}\right)$ be a rectangular M-metric space and $T$ be a self-mapping on $X$. If there exists $k \in[0,1)$ such that

$m_{r}(T x, T y) \leq k m_{r}(x, y)$ for all $x, y \in X$

and consider the sequence $\left\{x_{n}\right\}_{n \geq 0}$ defined by $x_{n+1}=T x_{n}$. If $x_{n} \rightarrow u$ as $n \rightarrow \infty$, then $T x_{n} \rightarrow$ Tu as $n \rightarrow \infty$.

Proof First, note that if $m_{r}\left(T x_{n}, T u\right)=0$, then $m_{r_{T x_{n}, T u}}=0$ and that is due to the fact that $m_{r_{T x_{n}, T u}} \leq m_{r}\left(T x_{n}, T u\right)$, which implies that

$m_{r}\left(T x_{n}, T u\right)-m_{r_{T x}, T u} \rightarrow 0$ as $\mathrm{n} \rightarrow \infty$ andthatis

$T x_{n} \rightarrow T u$ as $\mathrm{n} \rightarrow \infty$.

So, we may assume that $m_{r}\left(T x_{n}, T u\right)>0$, since by (4.1) we have $m_{r}\left(T x_{n}, T u\right)<m_{r}\left(x_{n}, u\right)$, then we have the following two cases:

If $m_{r}(u, u) \leq m_{r}\left(x_{n}, x_{n}\right)$, then it is easy to see that $m_{r}\left(x_{n}, x_{n}\right) \rightarrow 0$, which implies that $m_{r}(u, u)=0$, and since $m_{r}(T u, T u)<m_{r}(u, u)=0$, we deduce that $m_{r}(T u, T u)=$ $m_{r}(u, u)=0$, and $m_{r}\left(x_{n}, u\right) \rightarrow 0$; on the other, we have $m_{r}\left(T x_{n}, T u\right) \leq m_{r}\left(x_{n}, u\right) \rightarrow 0$.

Hence, $m_{r}\left(T x_{n}, T u\right)-m_{r_{X_{n}, T u}} \rightarrow 0$ and thus $T x_{n} \rightarrow T u$.

If $m_{r}(u, u) \geq m_{r}\left(x_{n}, x_{n}\right)$, and once again it is easy to see that $m_{r}\left(x_{n}, x_{n}\right) \rightarrow 0$, which implies that $m_{r_{x_{n}, u}} \rightarrow 0$. Hence, $m_{r}\left(x_{n}, u\right) \rightarrow 0$

and since $m_{r}\left(T x_{n}, T u\right)<m_{r}\left(x_{n}, u\right) \rightarrow 0$, we have $m_{r}\left(T x_{n}, T u\right)-m_{r_{T x_{n}, T u}} \rightarrow 0$ and thus $T x_{n} \rightarrow T u$ as desired.

Now we give some fixed point theorems.

Theorem 4.2 Let $\left(X, m_{r}\right)$ be a complete rectangular $M$ metric space and $T$ a self-mapping on $X$. If there exists $0<k<1$ such that
$m_{r}(T x, T y) \leq k m_{r}(x, y)$ for all $\mathrm{x}, \mathrm{y} \in \mathrm{X}$,

then $T$ has a unique fixed point $u$ in $X$, where $m_{r}(u, u)=0$.

Proof Let $x$ in $X$ be arbitrary. Using (4.2), we have

$m_{r}\left(T^{n} x, T^{n+1} x\right) \leq k m_{r}\left(T^{n-1} x, T^{n} x\right) \leq \cdots \leq k^{n} m_{r}(x, T x)$,

for all $n \geq 1$. We distinguish two cases.

Case 1 Let $T^{n} x=T^{m} x$ for some integers $n \neq m$. For example, take $m>n$. We have $T^{m-n}\left(T^{n} x\right)=T^{n} x$. Choose $y=T^{n} x$ and $p=m-n$. Then,

$T^{p} y=y$,

that is, $y$ is a periodic point of $T$. By (4.2) and (4.3), we have

$m_{r}(y, T y)=m_{r}\left(T^{p} y, T^{p+1} y\right) \leq k^{p} m_{r}(y, T y)$.

Since $k \in(0,1)$, we get $m_{r}(y, T y)=0$. On the other hand, we have

$$
\begin{aligned}
m_{r}(y, y)= & m_{r}\left(T^{p} y, T^{p} y\right) \leq k m_{r}\left(T^{p-1} y, T^{p-1} y\right) \\
& \leq \cdots<m_{r}(T y, T y) \leq k m_{r}(y, y)<m_{r}(y, y)
\end{aligned}
$$

Thus,

$m_{r}(y, y)=m_{r}(T y, T y)=0$.

Hence, $y=T y$, that is, $y$ is a fixed point of $T$.

Case 2 Suppose that $T^{n} x \neq T^{m} x$ for all integers $n \neq m$. We rewrite (4.3) as

$m_{r}\left(T^{n} x, T^{n+1} x\right) \leq k^{n} m_{r}(x, T x) \leq \frac{k^{n}}{1-k} m_{r}(x, T x)$.

Similarly, by (4.2), we have

$$
\begin{aligned}
& m_{r}\left(T^{n} x, T^{n+2} x\right) \leq k m_{r}\left(T^{n-1} x, T^{n+1} x\right) \leq \cdots \leq k^{n} m_{r}\left(x, T^{2} x\right) \\
& \quad \leq \frac{k^{n}}{1-k} m_{r}\left(x, T^{2} x\right) .
\end{aligned}
$$

Now, if $m>2$ is odd, then consider $m=2 p+1$ with $p \geq 1$. By (4.2) and (4.4), we have

$$
\begin{aligned}
m_{r}\left(T^{n} x, T^{n+m} x\right) & \leq m_{r}\left(T^{n} x, T^{n+1} x\right)+m_{r}\left(T^{n+1} x, T^{n+2} x\right) \\
& +\cdots+m_{r}\left(T^{n+2 p} x, T^{n+2 p+1} x\right) \\
& \leq k^{n} m_{r}(x, T x)+k^{n+1} m_{r}(x, T x)+\cdots \\
& +k^{n+2 p} m_{r}(x, T x) \\
& =k^{n} m_{r}(x, T x)\left[1+k+k^{2}+\cdots+k^{2 p}\right] \\
& \leq \frac{k^{n}}{1-k} m_{r}(x, T x) .
\end{aligned}
$$

On the other hand, if $m>2$ is even, then consider $m=2 p$ with $p \geq 2$. Again, by (4.2), (4.4) and (4.5), 


$$
\begin{aligned}
m_{r}\left(T^{n} x, T^{n+m} x\right) & \leq m_{r}\left(T^{n} x, T^{n+2} x\right)+m_{r}\left(T^{n+2} x, T^{n+3} x\right) \\
& +\cdots+m_{r}\left(T^{n+2 p-1} x, T^{n+2 p} x\right) \\
& \leq k^{n} m_{r}\left(x, T^{2} x\right)+k^{n+2} m_{r}(x, T x) \\
& +k^{n+3} m_{r}(x, T x)+\cdots+k^{n+2 p-1} m_{r}(x, T x) \\
& \leq k^{n} m_{r}\left(x, T^{2} x\right)+\frac{k^{n+2}}{1-k} m_{r}(x, T x) \\
& \leq k^{n} m_{r}\left(x, T^{2} x\right)+\frac{k^{n}}{1-k} m_{r}(x, T x) .
\end{aligned}
$$

We deduce from all cases that

$$
m_{r}\left(T^{n} x, T^{n+m} x\right) \leq k^{n} m_{r}\left(x, T^{2} x\right)+\frac{k^{n}}{1-k} m_{r}(x, T x)
$$

for all $n, m \geq 0$.

The right-hand side tends to 0 as $n \rightarrow \infty$, and since

$m_{r}\left(T^{n} x, T^{n+m} x\right)-m_{r^{n} x, T^{n+m_{x}}} \leq m_{r}\left(T^{n} x, T^{n+m} x\right)$,

we deduce that the sequence $\left\{T^{n} x\right\}$ is $m_{r}$-Cauchy in the $m_{r^{-}}$ complete rectangular $M$-metric space $\left(X, m_{r}\right)$. Hence, there exists some $u \in X$ such that

$\lim _{n \rightarrow \infty} m_{r}\left(T^{n} x, u\right)=\lim _{n, m \rightarrow \infty} m_{r}\left(T^{n} x, T^{m} x\right)=m_{r}(u, u)$.

In view (4.6), we get

$m_{r}(u, u)=\lim _{n \rightarrow \infty} m_{r}\left(T^{n} x, u\right)=\lim _{n, m \rightarrow \infty} m_{r}\left(T^{n} x, T^{m} x\right)=0$.

We shall prove that $T u=u$. Mention that we are still in case 2, that is, $T^{n} x \neq T^{m} x$ for all integers $n \neq m$. Now, we distinguish three subcases.

Subcase 1 If for all $n \geq 0, T^{n} x \notin\{u, T u\}$, the rectangular inequality implies that

$$
\begin{aligned}
m_{r}(u, T u) & \leq m_{r}\left(u, T^{n} x\right)+m_{r}\left(T^{n} x, T^{n+1} x\right)+m_{r}\left(T^{n+1} x, T u\right) \\
& \leq m_{r}\left(u, T^{n} x\right)+m_{r}\left(T^{n} x, T^{n+1} x\right)+k m_{r}\left(T^{n} x, u\right) .
\end{aligned}
$$

Taking limit as $n \rightarrow \infty$ and using (4.4) and (4.7), we get $m_{r}(u, T u)=0$ that is, $T u=u$.

Subcase 2 If there exists an integer $N$ such that $T^{N} x=u$. Due to case $2, T^{n} x \neq u$ for all $n>N$. Similarly, $T^{n} x \neq T u$ for all $n>N$. We reach subcase 1 , so $u$ is a fixed point of $T$.

Subcase 3 If there exists an integer $N$ such that $T^{N} x=T u$. Again, necessarily $T^{n} x \neq u$ and $T^{n} x \neq T u$ for all $n>N$. Similarly, we get $T u=u$.

We deduce that $u$ is a fixed point of $T$. To show the uniqueness of the fixed point $u$, assume that $T$ has another fixed point $v$. By (4.2),

$m_{r}(u, v)=m_{r}(T u, T v) \leq k m_{r}(u, v)$, which holds unless $m_{r}(u, v)=0$, so $u=v$.

Example 4.3 Taking $\theta=0$, we consider the rectangular $M$-metric space $\left(X_{0}, m_{r}\right)$ introduced in Example 2.4 where $X_{0}=[0, \infty)$ and $m_{r}(x, y)=\frac{x+y}{2}$ for all $x, y \in X_{0}$. Define the mapping $T$,

$$
\begin{aligned}
T: X_{0} & \rightarrow X_{0} \\
x & \mapsto \frac{x}{2} .
\end{aligned}
$$

Let $x, y \in[0, \infty)$, we have

$$
\begin{aligned}
& m_{r}(T x, T y)=m_{r}(x / 2, y / 2)=(x / 2+y / 2) / 2 \\
& =\frac{x+y}{4} \leq \frac{2}{3} \frac{x+y}{2} .
\end{aligned}
$$

Then, $T$ satisfies $m_{r}(T x, T y) \leq k m_{r}(x, y)$ with $0<k=\frac{2}{3}<1$.

Finally, all the conditions of Theorem 4.2 are satisfied. Therefore, $T$ has $u=0$ as a fixed point in $X_{0}$.

Theorem 4.4 Let $\left(X, m_{r}\right)$ be a complete rectangular $M$ metric space and $T$ be a self-mapping on $X$. If there exists $0<k<1$ such that

$m_{r}(T x, T y) \leq k \max \left\{m_{r}(x, y), m_{r}(x, T x), m_{r}(y, T y)\right\}$ for all $\mathrm{x}, \mathrm{y}$ $\in \mathrm{X}$

then $T$ has a unique fixed point $u$ in $X$, where $m_{r}(u, u)=0$.

Proof Let $x_{0} \in X$ and the sequence $\left\{x_{n}\right\}$ be defined as in the proof of Theorem 4.2. So, we may assume that $x_{n} \neq$ $x_{n+1}$ for all $n$.

For all natural number $n$, we have

$m_{n}=m_{r}\left(x_{n}, x_{n+1}\right)=m_{r}\left(T x_{n-1}, T x_{n}\right) \leq k \max \left\{m_{r}\left(x_{n}, x_{n+1}\right)\right.$, $\left.m_{r}\left(x_{n-1}, x_{n}\right)\right\}$.

Hence, if $\max \left\{m_{r}\left(x_{n}, x_{n+1}\right), m_{r}\left(x_{n-1}, x_{n}\right)\right\}=m_{r}\left(x_{n}, x_{n+1}\right)$, then inequality (8) implies

$m_{r}\left(x_{n}, x_{n+1}\right)<m_{r}\left(x_{n}, x_{n+1}\right)$

which leads to a contradiction. Therefore,

$\max \left\{m_{r}\left(x_{n}, x_{n+1}\right), m_{r}\left(x_{n-1}, x_{n}\right)\right\}=m_{r}\left(x_{n-1}, x_{n}\right)$ for all $\mathrm{n}$.

Thus, the sequence $\left\{x_{n}\right\}$ satisfies the hypothesis of Theorem 4.2. So, similarly to the proof of Theorem 4.2, we can easily deduce that $T$ has a unique fixed point $u$ in $X$, where $m_{r}(u, u)=0$.

Theorem 4.5 Let $\left(X, m_{r}\right)$ be a complete rectangular $M$ metric space and $T$ be a self-mapping on $X$. If there exists $0 \leq k<\frac{1}{2}$ such that 
$m_{r}(T x, T y) \leq k\left[m_{r}(x, T x)+m_{r}(y, T y)\right]$ for all $\mathrm{x}, \mathrm{y} \in \mathrm{X}$,

then Thas a unique fixed point $u$ in $X$, where $m_{r}(u, u)=0$.

Proof Let $x_{0} \in X$ and define the sequence $\left\{x_{n}\right\}$ by $x_{n}=T x_{n-1} \quad$ for all $\mathrm{n}=1,2, \ldots$

If there exists a natural number $k$ such that $x_{k}=x_{k+1}$, then $x_{k}$ is a fixed point of $T$. Indeed, we have

$x_{k}=T x_{k-1}=x_{k+1}=T x_{k}$

and $x_{k}$ is the desired point. Therefore, we can assume that $x_{n} \neq x_{n+1}$ for all $n$. By (4.11), we have

$m_{r}\left(x_{n}, x_{n+1}\right)=m_{r}\left(T x_{n-1}, T x_{n}\right) \leq k\left[m_{r}\left(x_{n-1}, x_{n}\right)+m_{r}\left(x_{n}, x_{n+1}\right)\right]$

and so

$m_{r}\left(x_{n}, x_{n+1}\right) \leq \frac{k}{1-k} m_{r}\left(x_{n-1}, x_{n}\right)=r m_{r}\left(x_{n-1}, x_{n}\right)$,

where $0 \leq r=\frac{k}{1-k}<1$. Then, by the completeness of $X$ and Lemma 3.5, we obtain $x_{n} \rightarrow x$ for some $x \in X$. Hence, we find

$\lim _{n \rightarrow \infty}\left[m_{r}\left(x_{n}, x\right)-m_{r_{x_{n}, \mathrm{x}}}\right]=0$ and $\lim _{\mathrm{n} \rightarrow \infty}\left[\mathrm{M}_{\mathrm{r}_{\mathrm{x}, \mathrm{x}}}-\mathrm{m}_{\mathrm{r}_{\mathrm{x}, \mathrm{x}}}\right]=0$

and since $m_{r_{x_{n}, x}} \rightarrow 0$ we have $m_{r}\left(x_{n}, x\right) \rightarrow 0$ and $M_{r_{x_{n}, x}} \rightarrow 0$.

By Remark 2.3, we get $m_{r}(x, x)=0=m_{r_{x, T x}}$ and by (4.11)

$m_{r}\left(x_{n+1}, T x\right)=m_{r}\left(T x_{n}, T x\right) \leq k\left[m_{r}\left(x_{n}, x_{n+1}\right)+m_{r}(x, T x)\right]$.

Using the fact $m_{r}\left(x_{n}, x_{n+1}\right) \rightarrow 0$, we get

$\underset{n \rightarrow \infty}{\limsup } m_{r}\left(x_{n+1}, T x\right)=\underset{n \rightarrow \infty}{\limsup } m_{r}\left(T x_{n}, T x\right) \leq k m_{r}(x, T x)$.

On the other hand,

$m_{r}(x, T x)-m_{r_{x, T x}} \leq m_{r}\left(x, x_{n}\right)+m_{r}\left(x_{n}, T x\right)$

implies that

$m_{r}(x, T x) \leq \limsup _{n \rightarrow \infty}\left[m_{r}\left(x, x_{n}\right)+m_{r}\left(x_{n}, T x\right)\right] \leq k m_{r}(x, T x)$

since $\quad m_{r_{x, T x}}=0 \quad$ and $\quad m_{r}\left(x_{n}, x\right) \rightarrow 0 . \quad$ Consequently, $m_{r}(x, T x)=0$. By contradiction (4.11), we have

$m_{r}(T x, T x) \leq k\left[m_{r}(x, T x)+m_{r}(x, T x)\right]=2 k m_{r}(x, T x)$

and so

$m_{r}(T x, T x)=0=m_{r}(x, x)=m_{r}(x, T x)$.

This shows that $x=T x$ by the condition $\left(R M_{1}\right)$. Uniqueness of the fixed point follows by (4.12). Assume that $T$ has two fixed points $u, v$. We have $m_{r}(u, v)=m_{r}(T u, T v) \leq k\left[m_{r}(u, T u)+m_{r}(v, T v)\right]=0$

and

$m_{r}(u, v)=0$.

Using the fact $m_{r}(u, u)=0=m_{r}(v, v)$, we get $u=v$ as required.

Theorem 4.6 Let $\left(X, m_{r}\right)$ be a complete rectangular $M$ metric space and $T$ be a self-mapping on $X$. If there exists $0 \leq k<\frac{\sqrt{3}-1}{2}$ such that

$m_{r}(T x, T y) \leq k\left[m_{r}(x, T y)+m_{r}(y, T x)\right]$,

for all $x, y \in X$, then $T$ has a unique fixed point $u$ in $X$, where $m_{r}(u, u)=0$.

Proof Suppose that $x_{0} \in X$ and $T^{n} x_{0}=x_{n}$. Now we show that

$m_{r}\left(x_{n}, x_{n+1}\right) \rightarrow 0$ as $\mathrm{n} \rightarrow \infty$.

Using inequality (4.12), we get

$m_{r}\left(x_{n}, x_{n}\right)=m_{r}\left(T x_{n-1}, T x_{n-1}\right) \leq 2 k m_{r}\left(x_{n-1}, x_{n}\right)$,

for all $n \in \mathbb{N}$. From the inequality (4.13) and the condition $\left(R M_{4}\right)$, we obtain

$$
\begin{aligned}
& m_{r}\left(x_{n}, x_{n+1}\right)=m_{r}\left(T x_{n-1}, T x_{n}\right) \leq k\left[m_{r}\left(x_{n-1}, x_{n+1}\right)+m_{r}\left(x_{n}, x_{n}\right)\right] \\
& \leq k\left[\begin{array}{c}
m_{r}\left(x_{n-1}, x_{n}\right)-m_{r_{x_{n-1}, x_{n}}}+m_{r}\left(x_{n}, x_{n}\right)-m_{r_{x_{n}, x_{n}}} \\
+m_{r}\left(x_{n}, x_{n+1}\right)-m_{x_{x_{n}, x_{n+1}}}+m_{r_{x_{n-1}, x_{n+1}}}+m_{r}\left(x_{n}, x_{n}\right)
\end{array}\right]
\end{aligned}
$$

If we take

$R_{n}=m_{r}\left(x_{n}, x_{n}\right)-m_{r_{x_{n-1}, x_{n}}}-m_{r_{x_{n}, x_{n+1}}}+m_{r_{x_{n-1}, x_{n+1}}}$,

then we get the following cases:

Case 1 Let us consider

$m_{r}\left(x_{n-1}, x_{n-1}\right) \leq m_{r}\left(x_{n}, x_{n}\right) \leq m_{r}\left(x_{n+1}, x_{n+1}\right)$.

Then, we get

$$
\begin{aligned}
& m_{r_{x_{n}, x_{n+1}}}=m_{r}\left(x_{n}, x_{n}\right), \mathrm{m}_{\mathrm{r}_{\mathrm{x}_{\mathrm{n}-1}, \mathrm{x}_{\mathrm{n}}}}=\mathrm{m}_{\mathrm{r}}\left(\mathrm{x}_{\mathrm{n}-1}, \mathrm{x}_{\mathrm{n}-1}\right), \mathrm{m}_{\mathrm{r}_{\mathrm{x}_{\mathrm{n}-1}, \mathrm{x}_{\mathrm{n}+1}}} \\
& \quad=m_{r}\left(x_{n-1}, x_{n-1}\right)
\end{aligned}
$$

and so

$R_{n}=0$.

Case 2 Let us consider

$m_{r}\left(x_{n+1}, x_{n+1}\right) \leq m_{r}\left(x_{n}, x_{n}\right) \leq m_{r}\left(x_{n-1}, x_{n-1}\right)$.

By the similar arguments used in Case 1, we get $R_{n}=0$.

Case 3 Let us consider 
$m_{r}\left(x_{n}, x_{n}\right) \leq m_{r}\left(x_{n+1}, x_{n+1}\right) \leq m_{r}\left(x_{n-1}, x_{n-1}\right)$.

Then, we get

$$
\begin{aligned}
& m_{r_{x_{n}, x_{n+1}}}=m_{r}\left(x_{n}, x_{n}\right), \mathrm{m}_{\mathrm{r}_{\mathrm{x}_{\mathrm{n}}-1}, \mathrm{x}_{\mathrm{n}}}=\mathrm{m}_{\mathrm{r}}\left(\mathrm{x}_{\mathrm{n}}, \mathrm{x}_{\mathrm{n}}\right), \mathrm{m}_{\mathrm{r}_{\mathrm{x}_{\mathrm{n}-1}, \mathrm{x}_{\mathrm{n}+1}}} \\
& \quad=m_{r}\left(x_{n+1}, x_{n+1}\right)
\end{aligned}
$$

and so

$R_{n}=m_{r}\left(x_{n+1}, x_{n+1}\right)-m_{r}\left(x_{n}, x_{n}\right) \leq m_{r}\left(x_{n+1}, x_{n+1}\right)$.

Case 4 Let us consider

$m_{r}\left(x_{n-1}, x_{n-1}\right) \leq m_{r}\left(x_{n+1}, x_{n+1}\right) \leq m_{r}\left(x_{n}, x_{n}\right)$.

By the similar arguments used in Case 3, we get

$R_{n}=m_{r}\left(x_{n}, x_{n}\right)-m_{r}\left(x_{n+1}, x_{n+1}\right) \leq m_{r}\left(x_{n}, x_{n}\right)$.

Case 5 Let us consider

$m_{r}\left(x_{n}, x_{n}\right) \leq m_{r}\left(x_{n-1}, x_{n-1}\right) \leq m_{r}\left(x_{n+1}, x_{n+1}\right)$.

By the similar arguments used in Case 3, we get

$R_{n}=m_{r}\left(x_{n-1}, x_{n-1}\right)-m_{r}\left(x_{n}, x_{n}\right) \leq m_{r}\left(x_{n+1}, x_{n+1}\right)$.

Case 6 Let us consider

$m_{r}\left(x_{n+1}, x_{n+1}\right) \leq m_{r}\left(x_{n-1}, x_{n-1}\right) \leq m_{r}\left(x_{n}, x_{n}\right)$.

By the similar arguments used in Case 3, we get

$R_{n}=m_{r}\left(x_{n}, x_{n}\right)-m_{r}\left(x_{n+1}, x_{n+1}\right) \leq m_{r}\left(x_{n}, x_{n}\right)$.

If $R_{n}=0$, then we obtain

$m_{r}\left(x_{n}, x_{n+1}\right) \leq k\left[m_{r}\left(x_{n-1}, x_{n}\right)+m_{r}\left(x_{n}, x_{n+1}\right)\right]$

and so

$m_{r}\left(x_{n}, x_{n+1}\right) \leq \frac{k}{1-k} m_{r}\left(x_{n-1}, x_{n}\right)$.

If $R_{n}<m_{r}\left(x_{n}, x_{n}\right)$, then using inequality (4.13), we obtain

$R_{n}<2 k m_{r}\left(x_{n-1}, x_{n}\right)$

and so

$m_{r}\left(x_{n}, x_{n+1}\right) \leq \frac{k(2 k+1)}{1-k} m_{r}\left(x_{n-1}, x_{n}\right)$.

If $R_{n}<m_{r}\left(x_{n+1}, x_{n+1}\right)$, then using inequality (4.13), we obtain

$R_{n}<2 k m_{r}\left(x_{n}, x_{n+1}\right)$

and so

$m_{r}\left(x_{n}, x_{n+1}\right) \leq \frac{k}{1-k(2 k+1)} m_{r}\left(x_{n-1}, x_{n}\right)$.

Since $0 \leq k<\frac{\sqrt{3}-1}{2}$, then we get $\frac{k}{1-k}<1, \frac{k(2 k+1)}{1-k}<1$, $\frac{k}{1-k(2 k+1)}<1$ and so using Lemma 3.5, we have $m_{r}\left(x_{n}, x_{n+1}\right) \rightarrow 0$

as $n \rightarrow \infty$. Using the completeness hypothesis, we obtain $x_{n} \rightarrow u$ for some $u \in X$ and so

$\lim _{n \rightarrow \infty}\left[m_{r}\left(x_{n}, u\right)-m_{r_{x_{n}, u}}\right]=0$ and $\lim _{\mathrm{n} \rightarrow \infty}\left[\mathrm{M}_{\mathrm{r}_{\mathrm{x}_{\mathrm{n}}, \mathrm{u}}}-\mathrm{m}_{\mathrm{r}_{\mathrm{x}_{\mathrm{n}}, \mathrm{u}}}\right]=0$.

Since $m_{r_{x_{n}, u}} \rightarrow 0$, we have $m_{r}\left(x_{n}, u\right) \rightarrow 0$ and $M_{r_{x_{n}, u}} \rightarrow 0$. By Remark 2.3, we get

$m_{r}(u, u)=0=m_{r_{u, T u}}$.

From inequality (4.12), we obtain

$$
\begin{aligned}
m_{r}(u, T u) \leq & \limsup _{n \rightarrow \infty} m_{r}\left(u, x_{n}\right)+\limsup _{n \rightarrow \infty}\left(x_{n}, T u\right) \\
= & \underset{n \rightarrow \infty}{\limsup m_{r}}\left(x_{n}, T u\right) \\
\leq & \limsup _{n \rightarrow \infty}\left(k\left[m_{r}\left(x_{n-1}, T u\right)+m_{r}\left(x_{n}, u\right)\right]\right) \\
\leq & \limsup _{n \rightarrow \infty} m_{r}\left(x_{n-1}, T u\right)+\underset{n \rightarrow \infty}{\lim \sup k m_{r}}\left(x_{n}, u\right) \\
\leq & \limsup _{n \rightarrow \infty}\left[m_{r}\left(x_{n-1}, u\right)-m_{r_{x_{n-1}, u}}+m_{r}(u, u)\right. \\
& \left.-m_{r_{u, u}}+m_{r}(u, T u)-m_{r_{u, T u}}\right] \leq k m_{r}(u, T u),
\end{aligned}
$$

which implies $m_{r}(u, T u)=0$ since $0 \leq k<\frac{\sqrt{3}-1}{2}$. Using inequality (4.13), we get

$0 \leq m_{r}(T u, T u) \leq 2 k m_{r}(u, T u)=0$

and so

$m_{r}(T u, T u)=m_{r}(u, T u)=m_{r}(u, u)$.

Using condition $\left(R M_{1}\right)$, we get $u=T u$. Now we prove that $u$ is a unique fixed point of $T$. Let us consider $u, v \in X$ with $u \neq v, T u=u$ and $T v=v$. Using the condition (4.12), we have

$$
\begin{aligned}
0<m_{r}(u, v) & =m_{r}(T u, T v) \leq k\left[m_{r}(u, T v)+m_{r}(v, T u)\right] \\
& =2 k m_{r}(u, v)<m_{r}(u, v),
\end{aligned}
$$

which is a contradiction. Therefore, $u=v$ and $T$ has a unique fixed point $u$ in $X$.

\section{An application to fixed-circle problem}

The notions of a circle and of a fixed circle on a rectangular $M$-metric space are defined as follows:

Let $r>0$ and $x_{0} \in X$. The circle $C_{x_{0}, r}^{m_{r}}$ with the center $x_{0}$ and the radius $r$ is defined by

$$
C_{x_{0}, r}^{m_{r}}=\left\{x \in X: m_{r}\left(x, x_{0}\right)-m_{r_{x, x_{0}}}=r\right\} .
$$


Let $C_{x_{0}, r}^{m_{r}}$ be a circle and $T: X \rightarrow X$ be a self-mapping. If $T x=x$ for any $x \in C_{x_{0}, r}^{m_{r}}$ then the circle $C_{x_{0}, r}^{m_{r}}$ is called as the fixed circle of $T$.

Now we give the following fixed-circle result.

Theorem 5.1 Let $\left(X, m_{r}\right)$ be a rectangular M-metric space and $C_{x_{0}, r}^{m_{r}}$ be any circle on $X$. Let us define the mapping

$\varphi: X \rightarrow[0, \infty), \varphi(x)=m_{r}\left(x, x_{0}\right)-m_{r_{x, x_{0}}}$,

for all $x \in X$. If there exists a self-mapping $T: X \rightarrow X$ satisfying

$\left(C M_{1}\right) \quad m_{r}(x, T x)-m_{r_{x, T x}} \leq \varphi(x)-\varphi(T x)$,

$\left(C M_{2}\right) \quad m_{r}\left(T x, x_{0}\right)-m_{r_{T x, x_{0}}} \geq r$

$\left(C M_{3}\right) \quad m_{r_{x, T x}}=M_{r_{x, T x}}$

for each $x \in C_{x_{0}, r}^{m_{r}}$, then the circle $C_{x_{0}, r}^{m_{r}}$ is a fixed circle of $T$.

Proof Let $x \in C_{x_{0}, r}^{m_{r}}$. Then, we have $m_{r}\left(x, x_{0}\right)-m_{r_{x, x_{0}}}=r$. Now we prove that $T x=x$ whenever $x \in C_{x_{0}, r}^{m_{r}}$. From the condition $\left(C M_{1}\right)$, we obtain

$$
\begin{aligned}
& m_{r}(x, T x)-m_{r_{x, T x}} \leq \varphi(x)-\varphi(T x) \\
& \quad=\left[m_{r}\left(x, x_{0}\right)-m_{r_{x, x_{0}}}\right]-\left[m_{r}\left(T x, x_{0}\right)-m_{r_{T x, x_{0}}}\right] .
\end{aligned}
$$

Using the conditions $\left(R M_{2}\right)$ and $\left(C M_{2}\right)$, we get

$m_{r}\left(T x, x_{0}\right)-m_{r_{T x, x_{0}}}=r$

and $T x \in C_{x_{0}, r}^{m_{r}}$. Using inequality (5.1), we obtain

$m_{r}(x, T x)-m_{r_{x, T x}}=0 \Rightarrow m_{r}(x, T x)=m_{r_{x, T x}}$.

From conditions (5.2), $\left(C M_{3}\right)$ and $\left(R M_{1}\right)$, we find

$m_{r}(x, T x)=m_{r_{x, T x}}=M_{r_{x, T x}} \Rightarrow x=T x$.

Consequently, the circle $C_{x_{0}, r}^{m_{r}}$ is a fixed circle of $T$.

Now we give an illustrative example.

Example 5.2 Let us consider the rectangular $M$-metric space $\left(X_{\frac{\pi}{2}}, m_{r}\right)$ introduced in Example 2.4, the circle $C_{2 i, 1}^{m_{r}}$ on $X_{\frac{\pi}{2}}$ and define the self-mapping $T: X_{\frac{\pi}{2}} \rightarrow X_{\frac{\pi}{2}}$ as

$T z=\left\{\begin{array}{ccc}i z & ; & |z|<4 \\ z & ; & |z| \geq 4\end{array}\right.$,

for all $z \in X_{\frac{\pi}{2}}$. Then, the self-mapping $T$ satisfies the conditions $\left(C M_{1}\right),\left(C M_{2}\right)$ and $\left(C M_{3}\right)$ for $x \in C_{2 i, 1}^{m_{r}}$ such that

$C_{2 i, 1}^{m_{r}}=\left\{x \in X_{\frac{\pi}{2}}: \frac{|x|+|2 i|}{2}-m_{r x, 2 i}=1\right\}=\{0,4 i\}$.

Clearly $C_{2 i, 1}^{m_{r}}$ is a fixed circle of $T$.

\section{Conclusion}

Let us consider Example 2.6 and the contractive condition given in Theorem 4.2. Then, we have

$m_{r}(T x, T y) \leq k m_{r}(x, y)$,

for all $x, y \in X$ and $k \in(0,1)$. Using the definition of $m_{r}$ defined in Example 2.6, we get

$$
\begin{gathered}
m_{r}(T x, T y)=\operatorname{md}(T x, T y)+n \leq k[m d(x, y)+n] \\
=k m d(x, y)+k n \\
\Rightarrow d(T x, T y) \leq k d(x, y)+\frac{n(k-1)}{m} .
\end{gathered}
$$

Inequality (6.1) does not satisfy the Banach contraction principle

$d(T x, T y) \leq k d(x, y)$,

for all $x, y \in X$ and $k \in(0,1)$ on a rectangular metric space. Therefore, it is important to study fixed-point theorems using different contractive conditions on a rectangular $M$ metric space even if a rectangular $M$-metric and a rectangular metric generate same topology. Furthermore, in the last section, we have given an introduction to the fixedcircle problem [8]. On this new space, it is possible to study some fixed-circle results by various aspects.

Open Access This article is distributed under the terms of the Creative Commons Attribution 4.0 International License (http://creative commons.org/licenses/by/4.0/), which permits unrestricted use, distribution, and reproduction in any medium, provided you give appropriate credit to the original author(s) and the source, provide a link to the Creative Commons license, and indicate if changes were made.

\section{References}

1. Arshad, M., Ahmad, J., Karapınar, E.: Some common fixed point results in rectangular metric spaces. Int. J. Anal. Art. ID 307234 (2013)

2. Asadi, M., Karapınar, E., Salimi, P.: New extension of $p$-metric spaces with some fixed-point results on $M$-metric spaces. J. Inequal. Appl. 2014, 18 (2014)

3. Branciari, A.: A fixed point theorem of Banach-Caccioppoli type on a class of generalized metric spaces. Publ. Math. Debrecen 57(1-2), 31-37 (2000)

4. George, R., Radenović, S., Reshma, K.P., Shukla, S.: Rectangular b-metric space and contraction principles. J. Nonlinear Sci. Appl. 8(6), 1005-1013 (2015)

5. Matthews, S.G.: Partial metric topology. In: Papers on General Topology and Applications (Flushing, NY, 1992). Ann. New York Acad. Sci., vol. 728, pp. 183-197. New York Acad. Sci., New York (1994)

6. Monfared, H., Azhini, M., Asadi, M.: Fixed point results on $M$ metric spaces. J. Math. Anal. 7(5), 85-101 (2016)

7. Shukla, S.: Partial rectangular metric spaces and fixed point theorems. Sci. World J. Article ID 756298 (2014)

8. Özgür, N.Y., Taş, N.: Some fixed-circle theorems on metric spaces. Bull. Malays. Math. Sci. Soc. (2017). https://doi.org/10. 1007/s40840-017-0555-z 